\title{
A COUNTEREXAMPLE FOR MAXIMAL OPERATORS OVER A CANTOR SET OF DIRECTIONS
}

\author{
Nets HaWK KaTZ
}

\begin{abstract}
A вstract. We produce a counterexample to the boundedness of the Cantor set maximal operator. We also produce a sharp counterexample for the restriction of the maximal operator of a truncated Cantor set to a strip.
\end{abstract}

\section{$\S 0$. Introduction}

Let $\mathcal{R}_{N}$ be the set of rectangles in $\mathbb{R}^{2}$ having length 1 and width $\frac{1}{N}$. Let us define the maximal operator on $\mathbb{R}^{2}$ given by

$$
\left(\mathcal{M}_{N} f\right)(x)=\sup _{x \in R \in \mathcal{R}_{N}} \frac{1}{|R|} \int_{R}|f|
$$

Cordoba in $[\mathrm{C}]$ proved that for any $f \in L^{2}\left(\mathbb{R}^{2}\right)$, one has the inequality,

$$
\left\|\mathcal{M}_{N} f\right\|_{L^{2}} \leq C \sqrt{1+\log N}\|f\|_{L^{2}} .
$$

In fact, he showed something stronger. He demonstrated that for any $S \in \mathcal{R}_{N}$ where $\chi_{S}(x)$ is the characteristic function of $S$ that

$$
\left\|\chi_{S} \mathcal{M}_{N}^{S} f\right\|_{L^{2}} \leq C \frac{\sqrt{1+\log N}}{\sqrt{N}}\|f\|_{L^{2}} .
$$

Here $\mathcal{M}_{N}^{S}$ is defined just as $\mathcal{M}_{N}$ by

$$
\left(\mathcal{M}_{N}^{S} f\right)(x)=\sup _{x \in R \in \mathcal{R}_{N}^{S}} \frac{1}{|R|} \int_{R}|f|
$$

where $\mathcal{R}_{N}^{S}$ is defined as the set of elements of $\mathcal{R}_{N}$ making an angle of at least $\frac{\pi}{4}$ with $S$. He proved (0.1) by observing that it is sufficient to work on a square and combining (0.2) on $N$ parallel rectangles $S$.

Received June 5, 1996.

The author was partially supported by a National Science Foundation Postdoctoral Fellowship. 
One line of research is to determine whether restrictions on the directions of allowed rectangles will improve the estimate (0.1). That is let $\Sigma \subset[0,1]$ and define $\mathcal{R}_{N}^{\Sigma}$ to be those elements of $\mathcal{R}_{N}$ having slope in $\Sigma$. Then we define

$$
\left(\mathcal{M}_{N}^{\Sigma} f\right)(x)=\sup _{x \in R \in \mathcal{R}_{N}^{\Sigma}} \frac{1}{|R|} \int_{R}|f| .
$$

It suffices to consider sets $\Sigma$ whose elements are fractions with denominator $N$. If $\Sigma$ is a lacunary set, then in particular it can have at most $C \log N$ elements. By a result of [NSW], one has that

$$
\left\|\mathcal{M}_{N}^{\Sigma} f\right\|_{L^{2}} \leq C\|f\|_{L^{2}}
$$

where the constant $C$ depends only on the order of lacunarity. Now, in light of the ideas in $[\mathrm{K}]$, (There, boundedness with constant $\sqrt{\log N}$ is obtained for a certain class of paraproducts whose boundedness implies boundedness of the maximal operator over unit intervals in $N$ directions. Boundedness of the same class of paraproducts multiplied by the scalar $\frac{1}{\sqrt{N}}$ implies boundedness of the maximal operator restricted to a strip) for any set $\Sigma$ with $\log N$ elements, one gets automatically

$$
\left\|\chi_{S} \mathcal{M}_{N}^{\Sigma} f\right\|_{L^{2}} \leq C \frac{\sqrt{1+\log \log N}}{\sqrt{N}}\|f\|_{L^{2}}
$$

but for $\Sigma$ lacunary, it is quite easy to obtain

$$
\left\|\chi_{S} \mathcal{M}_{N}^{\Sigma} f\right\|_{L^{2}} \leq C \frac{1}{\sqrt{N}}\|f\|_{L^{2}}
$$

where, in both cases, $S$ is parallel to the $y$-axis.

Now letting $N=3^{n}$ for some integer $n$, if $\Sigma$ is the truncated Cantor set (i.e. $\Sigma$ consists of all numbers of the form 0,1 , or $\sum_{j=1}^{n} \frac{a_{j}}{3^{j}}$ where $a_{j}$ is 0 or 2.), then it has been conjectured (e.g. [V]) that one obtains (0.3). Positive results for radial functions may be found in [DV]. However, we point out that (0.4) fails, i.e that

Theorem 1. Let $\Sigma$ be the Cantor set above, let $S$ be parallel to the y-axis. The exists a function $f$ and a constant $c>0$ so that

$$
\left\|\chi_{S} \mathcal{M}_{N}^{\Sigma} f\right\|_{L^{2}} \geq c \frac{\sqrt{\log N}}{\sqrt{N}}\|f\|_{L^{2}}
$$

Thus, in light of $[\mathrm{C}]$, the constant $\frac{\sqrt{\log N}}{\sqrt{N}}$ is sharp (as far as the exponent of $\log N$ for $\chi_{S} \mathcal{M}_{N}^{\Sigma}$.) We also prove the possibly less sharp 
Theorem 2. Let $\Sigma$ be as above. There exists a function $f$ and a constant c so that

$$
\left\|\mathcal{M}_{N}^{\Sigma} f\right\|_{L^{2}} \geq c \sqrt{\log \log N}\|f\|_{L^{2}}
$$

Theorem 2 also implies that for any $p<2$, there exists $c_{p}$ and a function $g$ so that

$$
\left\|\mathcal{M}_{N}^{\Sigma} g\right\|_{L^{p}} \geq c_{p}(\log \log N)^{\frac{1}{p}}\|f\|_{L^{p}}
$$

(In particular, we may choose $g=f^{\frac{2}{p}}$.) For $p>1+\frac{\log 2}{\log 3}$, this is a new result. However, for $p>2$, the problem of boundedness or unboundedness of $M_{N}^{\Sigma}$ in $L^{p}$ remains open.

\section{$\S 1$. Proof of Theorem 1}

It suffices to let $n>2$ for otherwise the theorem is trivial. It suffices to work on $S=\left[0,3^{-n}\right] \times\left[0, \frac{1}{3}\right]$. We define $Q(l)$ to be the square of sidelength $\frac{1}{N}$ described as $\left[0,3^{-n}\right] \times\left[(l-1) 3^{-n}, l 3^{-n}\right)$. For $1 \leq l \leq \frac{N}{3}$, we assign $s(l) \in$ $\Sigma$. We define the linear operator $L$ taking functions on $\mathbb{R}^{2}$ to functions on $S$ by

$$
L f(x)=\sum_{j=1}^{N} \chi_{Q_{j}}(x)\left(\frac{1}{\left|R_{j}\right|} \int_{R_{j}} f\right) .
$$

Here $R_{j}$ is the rectangle with dimensions $3 \times\left(\frac{1}{3 N}\right)$ centered at the center of $Q_{j}$ having slope $s(j)$. To prove the theorem, it suffices to show that there exists $f$, a function on $S$, a choice of $s(j)$ and a constant $c>0$ not depending on $N$ so that

$$
\left\|L L^{*} f\right\|_{L^{2}} \geq \frac{c \log N}{N}\|f\|_{L^{2}}
$$

We let $f=\chi_{S}$. Then $\|f\|_{L^{2}}=\frac{1}{\sqrt{N}}$. Thus we must show there exists a choice of $s(j)$ with

$$
\left\|L L^{*} f\right\|_{L^{2}} \geq \frac{c \log N}{N^{\frac{3}{2}}}
$$

Now by definition

$$
\left\|L L^{*} f\right\|_{L^{2}}=\left(\int_{S}\left(L L^{*} f\right)^{2}\right)^{\frac{1}{2}}
$$

whilst by Hölder's inequality,

$$
\int_{S} L L^{*} f \leq\left(\int_{S}\left(L L^{*} f\right)^{2}\right)^{\frac{1}{2}}\left(|S|^{\frac{1}{2}}\right)
$$


so that it suffices to show

$$
\int_{S} L L^{*} f \geq \frac{c \log N}{N^{2}}
$$

which is what we shall do.

Now,

$$
L^{*} f=\sum_{j=1}^{n}\left(\int_{Q_{j}} f\right) \frac{1}{\left|R_{j}\right|} \chi_{R_{j}} \geq c \frac{1}{N} \sum_{j=1}^{N} \chi_{R_{j}} .
$$

Thus for $x \in Q_{k}$, one has

$$
L L^{*} f(x) \geq c \sum_{j=1}^{N}\left|R_{j} \cap R_{k}\right|
$$

so that

$$
\int L L^{*} f \geq \frac{c}{N^{2}} \sum_{k=1}^{N} \sum_{j=1}^{N}\left|R_{j} \cap R_{k}\right| .
$$

Now whenever $|s(j)-s(k)| \geq \frac{|j-k|}{N}$, we have that

$$
\left|R_{j} \cap R_{k}\right| \geq \frac{c}{|s(j)-s(k)| N^{2}} .
$$

We let $K(j, k)=1$ when $|s(j)-s(k)| \geq \frac{|j-k|}{N}$ and $K(j, k)=0$ otherwise. Then it suffices to show there exists a choice of the map $s$ so that

$$
\sum_{j=1}^{N} \sum_{k=1}^{N} \frac{K(j, k)}{|s(j)-s(k)| N^{2}}=\frac{1}{N^{2}} \sum_{j=1}^{N} \sum_{k=1}^{N} \frac{K(j, k)}{|s(j)-s(k)|} \geq c n .
$$

We will find such a map $s$.

We produce a continuous version of $s$. Let $\mathfrak{C}$ be the middle thirds Cantor set contained in $[0,1]$. Let $d$ be the triadic distance on $[0,1]$. For any $f$ measurable taking $\left[0, \frac{1}{3}\right]$ to $\mathfrak{C}$, we define $K_{f}(x, y)=1$ when $|f(x)-f(y)| \geq$ $|x-y|$ and 0 otherwise. Clearly $K_{f}(x, y)$ cannot be 1 everywhere since then $f^{-1}$ would be a well defined Lipschitz map from $\mathfrak{C}$ onto $\left[0, \frac{1}{3}\right]$ which is impossible.

Proposition 1.1. There exists $f$ defined a.e. from $\left[0, \frac{1}{3}\right]$ to $\mathfrak{C}$ so that for every $n>0$,

$$
\int_{d(x, y) \geq 3^{-n}} \frac{K_{f}(x, y) d x d y}{|f(x)-f(y)|} \geq c n .
$$


Proof. Almost every $x \in\left[0, \frac{1}{3}\right]$ has a unique infinite ternary expansion $x=0.0 x_{1} x_{2} \ldots$ Every infinite ternary expansion $0 . y_{1} y_{2} \ldots$ denotes a unique element of $\mathfrak{C}$ as long as for every $j$, one has $y_{j}=0$ or $y_{j}=2$. We define

$$
f\left(0.0 x_{1} x_{2} \ldots\right)=. g\left(x_{1}\right) g\left(x_{2}\right) \ldots,
$$

where $g:\{0,1,2\} \longrightarrow\{0,2\}$ by $g(0)=g(1)=0$ and $g(2)=2$. For any $I$ triadic with $|I|=3^{-j}$, we observe by rescaling that

$$
\begin{gathered}
\int_{\left\{(x, y) \in I \times I: d(x, y)=3^{-j}\right\}} \frac{K_{f}(x, y) d x d y}{f(x)-f(y)}= \\
3^{1-j} \int_{\left\{(x, y) \in\left[0, \frac{1}{3}\right] \times\left[0, \frac{1}{3}\right]: d(x, y)=\frac{1}{3}\right\}} \frac{K_{f}(x, y) d x d y}{f(x)-f(y)} .
\end{gathered}
$$

On the other hand

$$
\int_{\{(x, y) \in[0,1] \times[0,1]: d(x, y)=1\}} \frac{K_{f}(x, y) d x d y}{f(x)-f(y)} \geq \frac{4}{81},
$$

since $\frac{1}{3} \leq|f(x)-f(y)| \leq 1$ when $x \in\left[0, \frac{2}{9}\right]$ and $y \in\left[\frac{2}{9}, \frac{1}{3}\right]$. Thus for any $l$, we have that

$$
\int_{d(x, y)=3^{-\imath}} \frac{K_{f}(x, y) d x d y}{|f(x)-f(y)|} \geq \frac{4}{81},
$$

by summing over all triadic $J \subset[0,1]$ with $|J|=3^{-l}$. Summing over $l$ going from 1 to $c \log N$, we obtain the proposition.

Now we are ready to define $s(j)$. If $j=\sum_{l=1}^{n-1} a_{l} 3^{n-l-1}$, then $s(j)=$ $\sum_{l=1}^{n} g\left(a_{l}\right) 3^{-l}$. By the same reasoning as the proposition, we prove (1.1) and Theorem 1.

\section{$\S 2$. Proof of Theorem 2}

We let $n>10$ for otherwise the theorem is trivial. We let the rectangles $R_{j}$ be as before. We define the function

$$
f=\sum_{j=1}^{3^{n-1}} \chi_{R_{j}} .
$$

We will prove the following three lemmas:

Lemma 2.1. With $C>0$ a universal constant independent of $N$,

$$
\int f^{2} \leq C \log N
$$


Lemma 2.2. There exists a constant $c>0$ independent of $N$, so that for all $1 \leq j \leq 3^{n-1}$,

$$
\frac{1}{\left|R_{j}\right|} \int_{R_{j}} f \geq c \log N
$$

Lemma 2.3. There exists a constant $c>0$ independent of $N$ so that

$$
\left|\cup R_{j}\right| \geq \frac{c \log \log N}{\log N}
$$

We first prove Theorem 2 from these three lemmas.

Proof of Theorem 2. By (2.2), we have that everywhere on $\cup R_{j}$,

$$
\mathcal{M}_{N}^{\Sigma} f \geq c \log N
$$

Now by (2.3) this yields

$$
\int_{\cup R_{j}}\left(M_{N}^{\Sigma} f\right)^{2} \geq c(\log \log N)(\log N)
$$

The inequalities (2.1) and (2.4) yield Theorem 2

Now we need only prove the three lemmas.

Proof of Lemma 2.1. By [C], the operator defined by

$$
(L g)(x)=\sum_{j=1}^{3^{n-1}} \chi_{Q_{j}}(x)\left(\frac{1}{\left|R_{j}\right|} \int_{R_{j}} g\right),
$$

is bounded on $L^{2}$ with norm less than or equal to $\frac{C \sqrt{\log N}}{N}$. Define the strip $S=\cup Q_{j}$. Then

$$
\left\|\chi_{S}\right\|_{L^{2}} \leq \frac{C}{\sqrt{N}}
$$

Thus

$$
\left\|L^{*} \chi_{S}\right\|_{L^{2}} \leq \frac{C \sqrt{\log N}}{N} .
$$

But $L^{*} \chi S=C \frac{1}{N} \sum_{j=1}^{3^{n-1}} \chi_{R_{j}}$, by definition. So that

$$
\|f\|_{L^{2}} \leq C \sqrt{\log N}
$$


which was to be shown.

Proof of Lemma 2.2. Observe that by definition,

$$
\frac{1}{\left|R_{j}\right|} \int_{R_{j}} f=c N \sum_{k=1}^{3^{n-1}}\left|R_{j} \cap R_{k}\right| .
$$

We let $d(s, t)$ denote the triadic distance between $s$ and $t$ for any $s, t \in[0,1]$ (i.e. $d(s, t)$ is the length of the smallest triadic interval containing both $s$ and $t$.) It suffices to show that for any $1 \leq l \leq n-1$, we have

$$
\sum_{\left\{k: d\left(\frac{j}{n}, \frac{k}{n}\right)=3^{-l}\right\}}\left|R_{j} \cap R_{k}\right| \geq \frac{c}{N} .
$$

For any $j$, there are $c 3^{n-l-2}$ numbers $k$ with $d\left(\frac{j}{N}, \frac{k}{N}\right)=3^{-l}$ and with $3^{-l} \leq|s(j)-s(k)| \leq 3^{1-l}$. These are all $k$ 's so that if the $l+1$ st digit of $\frac{j}{N}$ is 0 or 1 then the $l+1$ st digit of $\frac{k}{N}$ is 2 or vice versa. For each such $k$, we have that

$$
\left|R_{j} \cap R_{k}\right| \geq c \frac{3^{l}}{N^{2}}
$$

Summing over $3^{n-l-2}$ values, we obtain (2.5). Summing over $l$ yields the lemma.

Proof of Lemma 2.3. Let $D=\cup_{j=1}^{3^{n-1}} R_{j}$ and define the auxiliary functions

$$
t_{j}(x)=\frac{\chi_{R_{j}}(x)}{\sum_{k=1}^{3^{n-1}} \chi_{R_{k}}(x)},
$$

on $D$. Clearly, we have that

$$
\sum_{j=1}^{3^{n-1}} \int t_{j}(x) d x=|D|
$$

which is the quantity we wish to estimate. We define the set $R_{j}^{\lambda}$ to be the part of $R_{j}$ which is at distance approximately $\frac{1}{\lambda}$ from the $y$-axis. In other words,

$$
R_{j}^{\lambda}=\left\{\left(x_{1}, x_{2}\right) \in R_{j}: \frac{1}{\lambda} \leq\left|x_{1}\right| \leq \frac{2}{\lambda}\right\}
$$

We claim that

$$
\sum_{j=1}^{3^{n-1}} \int_{R_{j}^{\lambda}}\left(t_{j}(x)\right)^{-1} \leq C\left(\frac{1}{\lambda}+\frac{\log N}{\lambda^{2}}\right) .
$$


We may readily see that the left hand side of (2.6) is simply the same as

$$
\sum_{j=1}^{3^{n-1}} \sum_{k=1}^{3^{n-1}}\left|R_{j}^{\lambda} \cap R_{k}^{\lambda}\right|
$$

One immediately obtains that

$$
\sum_{j=1}^{3^{n-1}}\left|R_{j}^{\lambda}\right| \leq \frac{C}{\lambda}
$$

so that it suffices to show

$$
\sum_{j=1}^{3^{n-1}} \sum_{k \neq j}\left|R_{j}^{\lambda} \cap R_{k}^{\lambda}\right| \leq C\left(\frac{\log N}{\lambda^{2}}\right) .
$$

Now, we observe that $|s(j)-s(k)| \leq 3 d\left(\frac{j}{N}, \frac{k}{N}\right)$ so that the summand in $(2.7)$ is only nonzero for $(j, k)$ with

$$
d\left(\frac{j}{N}, \frac{k}{N}\right) \geq \frac{\lambda}{9}\left(\frac{|j-k|}{N}\right) .
$$

However for $\lambda$ sufficiently large (81 will suffice), one also has that (2.8) implies

$$
|s(j)-s(k)| \geq \frac{1}{3} d\left(\frac{j}{N}, \frac{k}{N}\right)
$$

The inequality (2.9) is true since $s$ maps the string 02 to 02 , while sending 10 to 00 , and 12 to 02 while sending 20 to 20 . Now we proceed to show (2.7). It suffices to show that for any fixed $l$,

$$
\sum_{j=1}^{3^{n-1}} \sum_{d\left(\frac{j}{N}, \frac{k}{N}\right)=3^{-l}}\left|R_{j}^{\lambda} \cap R_{k}^{\lambda}\right| \leq C\left(\frac{1}{\lambda^{2}}\right) .
$$

There are fewer than $\frac{C N 3^{n-l}}{\lambda^{2}}$ pairs $(j, k)$ satisfying $(2.8)$ with $d\left(\frac{j}{N}, \frac{k}{N}\right)=$ $3^{-l}$. However by $(2.9)$ each such pair contributes not more than $C \frac{3^{l}}{N^{2}}$ to the sum. Multiplying these two numbers gives (2.10) and summing over $l$ gives (2.7) and thus (2.6). 
From this point on, we restrict to $\lambda \leq \log N$ so that (2.6) becomes

$$
\sum_{j=1}^{3^{n-1}} \int_{R_{j}^{\lambda}}\left(t_{j}(x)\right)^{-1} \leq C\left(\frac{\log N}{\lambda^{2}}\right) .
$$

This means by Tchebycheff that for at least $\frac{N}{6}$ values of $j$ one has

$$
\int_{R_{j}^{\lambda}}\left(t_{j}(x)\right)^{-1} \leq 2 C\left(\frac{\log N}{N \lambda^{2}}\right) .
$$

From this we obtain for these $j$,

$$
\frac{1}{\left|R_{j}^{\lambda}\right|} \int_{R_{j}^{\lambda}}\left(t_{j}(x)\right)^{-1} \leq 2 C\left(\frac{\log N}{\lambda}\right),
$$

which implies by Jensen's inequality that

$$
\frac{1}{\left|R_{j}^{\lambda}\right|} \int_{R_{j}^{\lambda}}\left(t_{j}(x)\right) \geq c\left(\frac{\lambda}{\log N}\right),
$$

so that

$$
\int_{R_{j}^{\lambda}} t_{j}(x) \geq c\left(\frac{1}{N \log N}\right) .
$$

Summing over the $j$ 's for which (2.11) is valid, we obtain

$$
\sum_{j} \int_{R_{j}^{\lambda}} t_{j}(x) \geq c\left(\frac{1}{\log N}\right) .
$$

Now we restrict $\lambda$ to being a member of the set,

$$
\Lambda=\left\{2^{0}, 2^{1}, \ldots, 2^{\log \log N}\right\} .
$$

For fixed $j$ and any $\lambda_{1}, \lambda_{2} \in \Lambda$ with $\lambda_{1} \neq \lambda_{2}$ one has $R_{j}^{\lambda_{1}} \cap R_{j}^{\lambda_{2}}=\emptyset$. Now summing (2.12) over $\lambda \in \Lambda$, we obtain

$$
\sum_{j} \int t_{j} \geq c\left(\frac{\log \log N}{\log N}\right)
$$

which was to be shown. 


\section{References}

[C] A. Cordoba, The Kakeya maximal function and the spherical summation multipliers, Amer. J. Math. 99 (1977), 1-22.

[DV] J. Duoandikoetxea and A. Vargas, Directional operators and radial functions on the plane., Ark. Mat. 33 (1995), 281-291.

[K] N. Katz, Remarks on maximal operators over arbitrary sets of directions, Preprint.

[NSW] A. Nagel, E. Stein, and S. Wainger, Differentiation in lacunary directions, Proc. Nat. Acad. Sci. U.S.A. 75 (1978), 1060-1062.

[V] A. Vargas, A remark on a maximal function over a Cantor set of directions, Rend. Circ. Mat. Palermo 44 (1995), 273-282.

Department of Mathematics, Yale University, New Haven, CT 06520

E-mail address: katz-nets@math.yale.edu 